\title{
Influencia de la inteligencia emocional en el clima
}

\section{laboral}

\section{Influence of emotional intelligence in the work environment}

1 Evelyn Jahaira Aragundi Castro

(iD) https://orcid.org/ 0000-0002-5122-4640

Estudiante de la Maestría con trayectoria de Investigación en Gestión del Talento Humano, Instituto de Posgrado, Universidad Técnica de Manabí, Portoviejo, Ecuador earagundi3435@utm.edu.ec

2 Carlos Jimmy Piloso Rodríguez

https://orcid.org/0000-0003-3592-1750

Docente de la Universidad Técnica de Manabí, Instituto de Posgrado. Portoviejo, Ecuador. jimmy.piloso@utm.edu.ec

Artículo de Investigación Científica y Tecnológica

Enviado: $13 / 12 / 2021$

Revisado: $28 / 12 / 2021$

Aceptado: 28/01/2022

Publicado:05/02/2022

DOI: https://doi.org/10.33262/ap.v4i1.1.177

\section{Cítese: emocional en el clima laboral. AlfaPublicaciones, 4(1.1), 499-513. https://doi.org/10.33262/ap.v4i1.1.177}

Aragundi Castro, E. J., \& Piloso Rodríguez, C. J. (2022). Influencia de la inteligencia

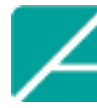

Ciencia Digital

ALFA PUBLICACIONES, es una Revista Multidisciplinar, Trimestral, que se publicará en soporte electrónico tiene como misión contribuir a la formación de profesionales competentes con visión humanística y crítica que sean capaces de exponer sus resultados investigativos y científicos en la misma medida que se promueva mediante su intervención cambios positivos en la sociedad. https://alfapublicaciones.com

La revista es editada por la Editorial Ciencia Digital (Editorial de prestigio registrada en la Cámara Ecuatoriana de Libro con No de Afiliación 663) www.celibro.org.ec

Esta revista está protegida bajo una licencia Creative Commons Attribution Non Commercial No Derivatives

4.0 International. Copia de la licencia: http://creativecommons.org/licenses/by-nc-nd/4.0/ 
Palabras

claves:

inteligencia

emocional, sentimientos, emociones, clima laboral.

\section{Keywords: \\ emotional intelligence, feelings, emotions, work environment.}

\section{Resumen}

La Inteligencia Emocional es definida como la capacidad que tienen las personas de poder entender y comprender los sentimientos tanto propios como de los demás, de poder motivarse y lograr manejar sus propias emociones tomando en consideración como estas pueden repercutir en los demás. El objetivo general de esta investigación se basa en analizar la influencia de la inteligencia emocional en el clima laboral. Asimismo, se utiliza una metodología basada en un diseño bibliográfico de tipo documental que permitirá conocer con mayor profundidad. Como conclusión se puede mencionar que La IE es necesaria en las organizaciones, y en especial en los trabajadores ya que esto facilita relacionarse entre ellos, transmiten conocimientos, y habilidades que pueden servirle a sus compañeros, y a su vez ser utilizados para alcanzar el éxito organizacional, cumpliendo metas y objetivos planteados dentro de la organización. Es importante tener claro que al tener personas en las organizaciones con inteligencia emocional incrementa la productividad, ya que existen personas creativas, con habilidades y emociones controladas dando paso a un clima laboral que genera un trabajo en equipo encaminado al éxito. Es necesario que los trabajadores en sus organizaciones sientan que cubren sus necesidades desde las más básicas a las de mayor jerarquía pues esto les permite sentir un estado de ánimo adecuado, estable, sin estrés laboral y en caso de que existieran exigencias mayores dentro de sus áreas de trabajo sean capaces de controlar estas presiones emocionales evitando posibles patologías que perjudiquen su calidad de vida, su entorno laboral y personal.

\section{Abstract}

Emotional Intelligence is defined as the ability that people have to be able to understand and comprehend their own feelings as well as those of others, to be able to motivate themselves and manage their own emotions taking into consideration how these can affect others. The general objective of this research is based on analyzing the influence of emotional intelligence on the work environment. Likewise, a methodology based on a documentary-type bibliographic design is used that will allow to know in greater depth. In conclusion, it can be mentioned that EI is necessary in organizations, and especially in workers, since this facilitates relationships between them, they transmit knowledge and skills that 
can serve their colleagues, and in turn be used to achieve organizational success, fulfilling goals and objectives set within the organization. It is important to be clear that having people in organizations with emotional intelligence increases productivity, since there are creative people, with skills and controlled emotions, giving way to a work environment that generates teamwork aimed at success. It is necessary for workers in their organizations to feel that their needs are covered from the most basic to those of the highest hierarchy, as this allows them to feel an adequate, stable state of mind, without work stress and in case there are greater demands within their areas of work are able to control these emotional pressures avoiding possible pathologies that harm their quality of life, their work and personal environment.

\section{Introducción}

A nivel mundial, todas las organizaciones se enfrentan a numerosos desafíos a nivel económico, político, social y aunado a estos se suma el recurso humano y cada aspecto que abarca este elemento fundamental dentro de cada empresa, que comprende la motivación, el bienestar y la satisfacción que estos tengan dentro de sus puestos de trabajo y en la empresa.

Por ello es fundamental lograr un clima organizacional adecuado donde prime lo anteriormente expuesto, y que la influencia de estos aspectos sea positiva en cada uno de los empleados para que estos sean capaces de realizar sus actividades conforme a los objetivos establecidos dentro de la organización.

El clima laboral según menciona Yslado et al. (2021), es la manera como son percibidas las características de una empresa y que estas pueden ser medibles, y que no necesariamente son iguales en todas las empresas, lo que permite poder determinar y ejecutar estrategias que puedan mejorar la situación en caso de que el clima no sea el mas idóneo o en caso contrario permite mantener una evaluación constante del clima estable presente en la organización.

Asimismo, Zhañay (2017) expone que el clima organizacional es la forma como los trabajadores se comportan en su trabajo y cómo interactúan con el entorno, por lo que el ambiente influye directamente en la percepción de cada individuo al igual que las diferentes percepciones que tienen cada uno de los trabajadores.

Como ya se ha mencionado el clima organizacional está relacionado con la manera en que el empleado se siente dentro de la empresa, por lo que en el inciden una serie de 
factores desde emocionales, así como físicos que definitivamente influyen directamente en el comportamiento de cada uno de los empleados y que afecta la productividad empresarial (Pilligua \& Arteaga, 2019). Además, es importante conocer de primera mano el comportamiento de cada empleado y como percibe su trabajo, su esfuerzo y si este es retribuido de manera adecuada.

En este sentido Pilligua \& Arteaga (2019) en su investigación mencionan que el primero en analizar el clima organizacional fue Elton Mayo alrededor de los años 1920 en una fabrica textil de Estados Unidos, donde analizó el comportamiento de los trabajadores de un área de la empresa, donde existía una rotación excesiva de trabajadores y cuyo comportamiento era irritantes, abatidos y desconsolados, sin una razón aparente a pesar de obtener beneficios económicos, que los conllevaba a tomar decisiones impulsivas de renunciar a sus puestos de trabajo.

De esta manera el clima organizacional depende directamente en aspectos psicológicos y organizacionales que deben ser analizados de forma individual y grupal para conocer los aspectos positivos y negativos que pueden incidir en este, de allí radica la fuerza del clima laboral como indican (Ramos \& Tejera, 2017).

En este sentido, Pilligua \& Arteaga (2019) mencionan una serie de factores que consideran permiten evaluar el clima laboral de una organización y se muestran en la tabla 1.

\section{Tabla 1}

Factores para evaluar el clima organizacional dentro de una organización

\begin{tabular}{cl}
\hline \multicolumn{1}{c}{ FACTORES } & \multicolumn{1}{c}{ DESCRIPCIÓN } \\
\hline & La comunicación dentro de las organizaciones debe ser \\
& mediante sistema abierto, significa que, no debe ser \\
& restringida por la estructura jerárquica de nivel \\
& descendente sino en forma horizontal, de esta forma, los \\
trabajadores conocerán los objetivos que persigue la & empresa, las necesidades y los logros obtenidos, de esta \\
Comunicación & manera permite influir en los individuos, ejercer un \\
& poder o credibilidad y de cierto modo, tener autoridad \\
& para liderar. \\
\hline Colaboración & Se evalúa el grado de madurez, el respeto, la manera de \\
& comunicarse, el grado de colaboración y compañerismo \\
& existente, y la confianza, siendo factores que suman \\
& importancia en el buen ambiente de trabajo. \\
\hline El liderazgo tiene sus teorías o enfoques, algunos de \\
ellos muy precisos, pero en el fondo son una \\
transformación para la empresa u organización; el \\
liderazgo en sí, involucra al personal que dirige y \\
aquellos que tienen responsabilidades en cada área.
\end{tabular}


Tabla 1

Factores para evaluar el clima organizacional dentro de una organización (continuación)

\begin{tabular}{ll}
\hline FACTORES & \multicolumn{1}{c}{ DESCRIPCIÓN } \\
\hline Se relaciona con el nivel de preparación académica, \\
habilidades y destrezas que tengan los trabajadores para \\
optar por un ascenso laboral, el mismo que repercute en \\
mejor calidad de vida, confort, sueldos considerables y \\
puestos acordes al desempeño, que se reflejen en la \\
evaluación de desempeño laboral. \\
\hline Es el conjunto de actitudes generales del individuo hacia \\
su trabajo. Probablemente, el empleado que se encuentre \\
dentro de un ambiente agradable en la organización, que \\
sus directivos reconozcan su trabajo y que lo incentiven \\
a seguir mejorando mediante ascensos u otro tipo de \\
motivaciones, generará satisfacción personal. \\
\hline Con relación a este aspecto, a través de las \\
investigaciones de Elton Mayo, pudo concluir que, los \\
ambientes, la iluminación, la reducción de ruidos, \\
herramientas y equipos para los empleados eran \\
elementos sustanciales (condiciones físicas), y que toda \\
organización debe brindar a sus trabajadores para que \\
sean más productivos. Así, en las organizaciones, los \\
directivos deben tomar decisiones acertadas, que \\
generan un excelente clima laboral. \\
\hline Condiciones físicas
\end{tabular}

Fuente: Pilligua \& Arteaga (2019)

En base a lo anteriormente expuesto es importante destacar que un clima organizacional negativo repercute en el rendimiento y en estado de ánimo de los empleados por lo que Zhañay (2017) menciona una serie de elementos que pueden generar desmotivación dentro de las organizaciones y se muestran en la tabla 2

Tabla 2

\section{Elementos desmotivadores dentro de una organización}

\begin{tabular}{ll}
\hline & Listado \\
\hline Salario insuficiente & Injusticias \\
\hline falta de horizontes & Despotismo \\
\hline Desconfianza, recelo y agresividad & Exceso de burocracia \\
\hline Mala educación & Falta de autonomía en el trabajo \\
\hline Rivalidades & Falta de interés hacia los trabajadores \\
\hline Crítica no positiva & La rutina del trabajo \\
\hline
\end{tabular}

Fuente: Zhañay (2017) 
Evaluar el clima organizacional y todo lo que esto representa es un tema de estudio profundo que realizan las empresas para lograr un mejoramiento continuo, que permita alcanzar, los objetivos de la empresa, mejorar las condiciones de los empleados, una satisfacción laboral, lo que se traduce en trabajadores productivos, motivados y con emociones controladas. Todos estos aspectos tienen una relación muy estrecha con la Pirámide de las necesidades humanas de Maslow (ver figura 1), donde Ferragud (2019) expone que si los individuos no satisfacen esas necesidades pueden manifestar sentimientos de frustración y de enojo por no poder alcanzar sus objetivos generando daños físicos y psíquicos que repercuten en sus actividades laborales y personales.

\section{Figura 1}

Pirámide de las necesidades humanas de Maslow

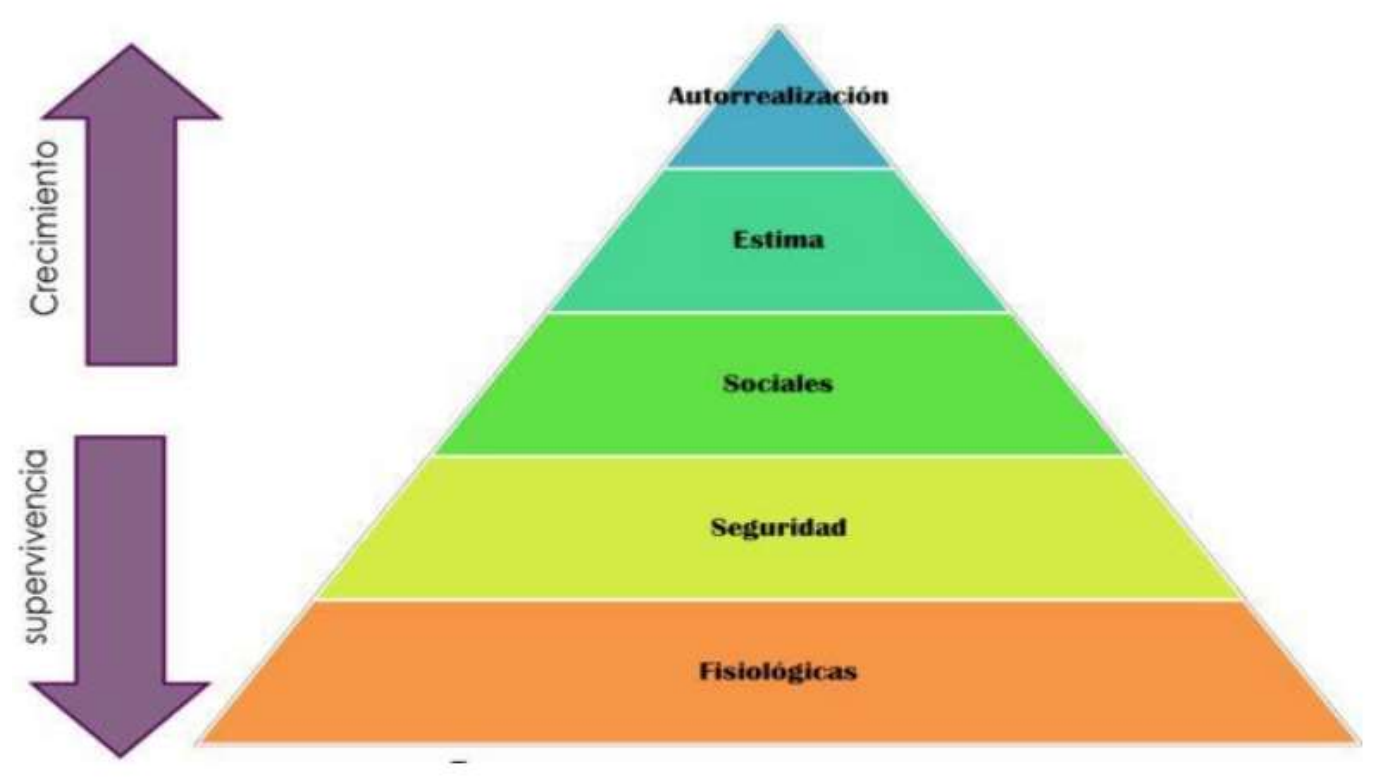

Fuente: Ferragud (2019)

En referencia a la pirámide de Maslow se menciona que las necesidades del nivel más bajo deben ser satisfechas hasta que el individuo se siente conforme con esto para poder avanzar a los niveles más altos, sin sentir inconformidad o insatisfacción de cada una de las necesidades puestos repercute en el comportamiento del ser humano y distorsiona el reconocimiento propio a sus capacidades o hacia el grupo de trabajo (Ferragud, 2019).

En este sentido esta investigación se basa en analizar la influencia de la inteligencia emocional en el clima laboral. Asimismo, se utiliza una metodología basada en un diseño bibliográfico de tipo documental que permitirá conocer con mayor profundidad estos aspectos antes mencionados. 


\section{Metodología}

La metodología utilizada en esta investigación se basa en un diseño bibliográfico utilizando procedimientos lógicos y mentales que permiten analizar, sintetizar información que permita el desarrollo de esta investigación (Palella \& Martins, 2010).

El tipo de investigación utilizado es el documental que se encarga de recopilar la información necesaria de diversas fuentes para lograr los objetivos de la investigación (Palella \& Martins, 2010).

\section{Resultados y Discusión}

\section{Inteligencia Emocional, evolución y teorías científicas}

Los pasos agigantados de la sociedad y las empresas con el propósito de evolucionar y no quedar atrapados en los avances que surgen producto de la globalización, hacen que las personas adopten estructuras emocionales con el fin de poder aumentar la productividad y que cada individuo sea eficiente en sus puestos de trabajo, dejando a un lado el impacto emocional que esto pueda generar en sus trabajadores (Carmona-Fuentes \& Rosas-Reyes, 2017).

Por tanto, es importante mencionar que, la inteligencia emocional en sus inicios fue estudiada como la inteligencia personal, la cual se basaba en estudiar las percepciones de una persona, y poder entender sus estados de ánimo, sus temperamentos, y objetivos dentro de la organización.

En este sentido otros autores acerca del comportamiento y las inteligencias múltiples, emprendieron una investigación basándose en diversas fuentes, que le permitieron poder aportar información relevante acerca de aspectos importantes para la ciencia cognitiva y la neurociencia citado por (Carmona-Fuentes \& Rosas-Reyes, 2017).

De esta manera, se establecieron las Teorías científicas de Inteligencia emocional, y la primera de ellas es la Teoría de las Inteligencias Múltiples, donde la inteligencia estaba conformada por una serie de aptitudes que les permitían a los individuos simplificar los problemas o transformar beneficios (Sanchez, 2019).

También, la Teoría de la efectividad es llamada como la teoría de los sentimientos, y es que está conformada por algo tan complejo como la afectividad, que es la que orienta la vida de las personas, y está conformada por dos estilos, el altruista donde el individuo se dedica a realizar su trabajo de forma egoísta y muy individual, y la que se encarga y preocupa de su familia, y su trabajo es decir se encarga de sus cosas (Sanchez, 2019). Además, en esta teoría están relacionados varios subsistemas relacionados con los sentimientos como los impulsos, el afecto y las emociones y que son bastante complejos 
pues estos sentimientos no se manifiestan igual en todas las personas sin embargo pueden ser estudiados uno por uno de forma separada.

De esta manera se pudo establecer ocho tipos de inteligencia y estas son:

- Inteligencia Lingüística

- Inteligencia Lógico-Matemática.

- Inteligencia Musical.

- Inteligencia Espacial

- Inteligencia kinestésico-corporal

- Inteligencia intrapersonal

- Inteligencia Natural

- Inteligencia espiritual

- Inteligencia existencial

De todas estas inteligencias la interpersonal es la que actualmente es denominada Inteligencia emocional y es la que permite estudiar el comportamiento de las personas dentro de las empresas, de manera que pueda alcanzar un comportamiento adecuado y eficaz en las actividades laborales lo que traerá consigo aspectos positivos en las organizaciones (Carmona-Fuentes \& Rosas-Reyes, 2017).

Asimismo, Duque et al. (2017) define la Inteligencia Emocional (IE) como la capacidad que tienen las personas de poder entender y comprender los sentimientos tanto propios como de los demás, de poder motivarse y lograr manejar sus propias emociones tomando en consideración como estas pueden repercutir en los demás.

Ahora bien, las emociones influyen en gran medida en el estado de ánimo de las personas que también puede repercutir en el estado físico, y que es observable en el comportamiento del individuo en un determinado tiempo. Estas emociones son parte de la persona y se ve reflejada en la manera en que interactúa con el resto de sus compañeros los cuales dependiendo de esas emociones pueden calificarlas como positivas o negativas (Duque et al., 2017). Por tanto, que, allí es donde tiene cabida la inteligencia emocional que permite a la persona poder controlar sus emociones pues estas pueden surgir una sobre otra y allí radica la habilidad de poder contralarla, capacidad que permite un buen desempeño laboral.

Factores de la IE

Para Duque (2006 citado por Guerrero, 2017) la IE posee tres factores clasificados según se muestra en la tabla 3. 


\section{Tabla 3}

Factores de la IE

\begin{tabular}{ll}
\hline FACTOR & DESCRIPCION \\
\hline \multirow{2}{*}{ Percepción de la emoción } & $\begin{array}{l}\text { Es la capacidad que tienen las personas para poder } \\
\text { reconocer sus emociones, de acuerdo a las } \\
\text { expresiones en su rostro, los diseños gráficos y los } \\
\text { relatos }\end{array}$ \\
\hline \multirow{2}{*}{$\begin{array}{l}\text { Comprensión de la emoción } \\
\text { Regulación }\end{array}$} & $\begin{array}{l}\text { Es la capacidad que poseen las personas para } \\
\text { reconocer como las emociones pueden cambiar con el } \\
\text { emociones }\end{array}$ \\
& $\begin{array}{l}\text { paso del tiempo, de manera que ayudan a poder } \\
\text { predecirlas y entender cómo se mezclan entre si }\end{array}$ \\
\hline \multirow{2}{*}{ las } & $\begin{array}{l}\text { Este factor consiste en determinar estrategias que } \\
\text { permitan poder controlar las emociones cuando el } \\
\text { individuo debe hacer frente a ellas. }\end{array}$ \\
\hline
\end{tabular}

Fuente: Guerrero (2017)

Asimismo, existen diversos factores que permiten que la IE se desarrolle y se fortalezca como las conversaciones, las reuniones laborales, los lemas, la lectura entre otros y aunado a ellas, establece que existen aún más factores como los emocionales, la salud hasta el nivel educativo que posea la persona (Guerrero, 2017).

Por otro lado, Roa (2019), menciona que la inteligencia de las personas va mas allá de su coeficiente intelectual y su nivel educativo, sino que deben ser capaces de poseer habilidades sociales y prácticas que les permita manejar situaciones, que mas allá de necesitar conocimientos para ello controlen emociones, sentimientos y estado de ánimos que deban enfrentar durante su jornada laboral.

\section{¿Por qué es importante la IE?}

En este mundo tan complejo, es importante poder controlar las emociones, y a nivel organizacional no es la excepción, pues las empresas siempre están en la búsqueda de personal que sea capaz, mas allá de ejecutar de forma eficaz sus actividades, puedan tener la habilidad de ser líderes, flexibles, que trabajen en grupo y puedan interrelacionarse con sus compañeros evitando la menor cantidad de conflictos posibles, ya que de esto depende un clima laboral armonioso que se vea reflejado en altos niveles de productividad.

Además, cuando las personas no pueden controlar las emociones pueden llegar a demostrar ser irritables, enojados y esto se puede ver reflejando en sus actividades laborales lo que provoca descontento por parte de sus compañeros o de los clientes. Por cuanto es importante que las personas sean capaces de reconocer cuáles son sus emociones, y la de los demás para poder ser capaces de establecer relaciones (Figueroa \& Jiménez, 2018). 
Como ya se ha mencionado la IE tiene un impacto directo con el clima laboral que a su vez se ve reflejado en la capacidad de motivación del personal y la iniciativa para ser personas más productivas dentro de su área de trabajo, por tanto, es necesario destacar que al momento de seleccionar personal se debe escoger personas emocionalmente competentes.

Sin, embargo esto no siempre se cumple, pues el trabajo y la falta de liderazgo o interés por parte de la empresa de mantener esta competencia emocional en sus empleados pueden conllevar a generar la pérdida de estabilidad emocional en los trabajadores. Por ello Figueroa \& Jiménez (2018) mencionan que las empresas deben estar siempre capacitando a su personal para que sean capaces de manejar el estrés y sus emociones considerando los siguientes aspectos:

- Aumentar el control sobre su propia salud

- Mejorar su bienestar físico, psicológico y socia

Al lograr controlar estos aspectos las empresas pueden obtener como beneficios mejorar la productividad lo que se traduce en ser mejor que la competencia y esto trae consigo el aumento de su desarrollo económico y social.

\section{La IE dentro de las empresas y sus aplicaciones}

Las personas pasan la mayor parte del día en sus actividades laborales, por cuanto este lugar es fundamental para propiciar relaciones entre los individuos, y es considerada como un sistema orgánico dependiente de la relación que exista entre sus componentes humanos, ya que a través de esta interacción se pueden negociar intereses o resolver conflictos, así como crear un espíritu de equipo (Gamboa, 2018).

Las empresas han descubierto que el éxito está directamente relacionado con la IE y que esta a diferencia del Coeficiente intelectual y cualquier otra medida de inteligencia se puede aumentar significativamente. La IE permite elevar la manera en que los trabajadores perciben el clima dentro de la organización mucho más que otros aspectos como la habilidad mental, la inteligencia y la experiencia (Gamboa, 2018).

Por ello es importante seleccionar capital humano emocionalmente inteligente, que a pesar de que es un poco difícil de detectar es un aspecto que marca la diferencia en las organizaciones, pero al estar frente a personas con IE alto estos son capaces de tomar decisiones mas acertadas, pues las personas que toman decisiones con emociones negativas pueden traer consecuencias graves (Apaza \& Flores, 2019). 


\section{La inteligencia emocional y su relación con la salud}

La Sociedad Española de Especialistas en Medicina del Trabajo mencionan que si no se optimiza la IE esta puede derivar en diversas patologías que no solo afectara a la persona que lo padece, sino que también repercute en el estado de ánimo de sus compañeros al alterar sus emociones y preocupaciones por la persona afectada (Figueroa \& Jiménez, 2018).

En este sentido, Figueroa \& Jiménez (2018), menciona que el estrés laboral es una de las afecciones más frecuentes en las organizaciones, y puede manifestarse con diversos síntomas a nivel cardiovascular, como taquicardias, aumento de la tensión arterial, también se pueden manifestar trastornos gastrointestinales y lesiones a nivel muscular como contracturas musculares. Esta serie de patologías pueden presentarse de forma individual o un cúmulo de ellas, lo que puede generar comportamientos no acordes al área de trabajo que derivan en ausentismo.

Existen ambientes laborales donde las exigencias son mayores y con riesgo más alto de presentar estrés laboral, de allí que las empresas siempre deben estar en la búsqueda de mejoramiento de sus empleados en cuanto al manejo de la IE para lograr un bienestar psicológico, ya que al presentar estas situaciones más allá de un clima laboral adverso, menciona Veliz et al. (2018), existe la posibilidad de errores mayores como eventos adversos, errores en la administración, malos tratos hacia el resto de los compañeros e inclusive sus superiores que no le permite cumplir con las funciones de su área de trabajo.

\section{Clima Organizacional y la IE}

El autor Gamboa (2018) en su Trabajo de grado de la Fundación Universitaria Los Libertadores sobre la Influencia de la Inteligencia Emocional en el mejoramiento del clima organizacional y reducción de índices de ausentismo laboral en M-MATEC S.A.S, menciona que en estudios realizados se demuestran que las personas con una IE mas alta son más conscientes de las emociones y que son capaces de manejarlas de mejor manera, lo que permite poder afrontar posibles situaciones de estrés que incide en un nivel de bienestar mayor, y por tanto dentro de la empresa tendrá mayor rendimiento laboral.

Las emociones influyen en gran medida en el comportamiento de todos los miembros de la empresa, y este "contagio emocional" es muy importante, pues si se presentan emociones negativas en una persona del grupo existen altas probabilidades que el resto del grupo se contagie de esa negatividad, asimismo, si la persona demuestra positivismo el resto del grupo se contagiara de esa actitud positiva (Aguayo, 2017).

De esta manera al ser los colaboradores los protagonistas del desarrollo empresarial y del éxito o fracaso que esta pueda tener, es importante que su clima laboral sea el más acorde posible, y como menciona Ramírez \& Zavaleta (2018) que cuando el individuo desarrolla 
habilidades inteligentemente emocionales la utiliza como herramientas que le permite lograr un mejor desempeño, ya que le facilita desarrollar la creatividad, motivación y otras habilidades que se relacionan con el éxito.

\section{Conclusiones}

- La IE es necesaria en las organizaciones, y en especial en los trabajadores ya que esto facilita relacionarse entre ellos, transmiten conocimientos, y habilidades que pueden servirle a sus compañeros, y a su vez ser utilizados para alcanzar el éxito organizacional, cumpliendo metas y objetivos planteados dentro de la organización.

- Es importante tener claro que al tener personas en las organizaciones con inteligencia emocional incrementa la productividad, ya que existen personas creativas, con habilidades y emociones controladas dando paso a un clima laboral que genera un trabajo en equipo encaminado al éxito.

- Es necesario que los trabajadores en sus organizaciones sientan que cubren sus necesidades desde las más básicas a las de mayor jerarquía pues esto les permite sentir un estado de ánimo adecuado, estable, sin estrés laboral y en caso de que existieran exigencias mayores dentro de sus áreas de trabajo sean capaces de controlar estas presiones emocionales evitando posibles patologías que perjudiquen su calidad de vida, su entorno laboral y personal.

\section{Referencias Bibliográficas}

Aguayo, G. (2017). La inteligencia emocional y el clima laboral de la Compañía Limitada TCAUDIT Trujillo \& Asociados firma auditora. Quito, Ecuador: Trabajo especial de grado de la Universidad Central del Ecuador para optar al título de Psicóloga Industrial.

Apaza, M., \& Flores, S. (2019). Inteligencia emocional en el comportamiento de las organizaciones. Lima, Perú: Trabajo especial de grado de la Universidad Peruana Unión.

Carmona-Fuentes, P., \& Rosas-Reyes, R. (2017). Influencia de la inteligencia emocional en el desempeño laboral. Revista LIDER 19(31), 107-118.

Duque, J., García, M., \& Hurado, A. (2017). Influencia de la inteligencia emocional sobre las competencias laborales: un estudio empírico con empleados del nivel administrativo. Estudios Gerenciales, 1-11.

Ferragud, C. (2019). Propuestas para mejorar el clima laboral y resolución de situaciones conflictivas. Trabajo especial de grado de la Universidad Siglo 21 para optar al título de Licenciatura en Gestión de Recursos Humanos. 
Figueroa, O., \& Jiménez, J. (2018). Inteligencia emocional y su impacto en las interrelaciones personales, la salud y la productividad laboral. Trabajo especial de Grado de la Corporación Universitaria Minuto de Dios.

Gamboa, G. (2018). Influencia de la inteligencia emocional en el mejoramiento del Clima Organizacional y reducción de índices de ausentismo laboral en M-Matec S.A.S. Bogotá, Colombia: Trabajo especial de grado de la Fundación Universitaria los Libertadores.

Guerrero, J. (2017). La inteligencia emocional y el desempeño laboral en los empleados del Ministerio de Trabajo y Promoción del Empleo. Lima, Perú: Trabajo especial de grado de la Universidad César Vallejo para optar al título de Maestro en Gestión Publica.

Palella, S., \& Martins, F. (2010). Metodología de la Investigación Cuantitativa. Carcas, Venezuela: FEDUPEL, Fondo Editorial de la Universidad Pedagógica Experimental Libertador.

Pilligua, C., \& Arteaga, F. (2019). El clima laboral como factor clave en el rendimiento productivo de las empresas estudio casp: Harpedex Cía. Ltda. Cuadernos Latinoamericanos de Administración XVI (28).

Ramírez, J., \& Zavaleta, V. (2018). Inteligencia emocional y desempeño laboral en una empresa constructora privada. 2017. Revista CIENCIA Y TECNOLOGÍA 14(4), 67-79.

Ramos, V., \& Tejera, E. (2017). Estudio de relaciones entre cultura, clima y fuerza de clima laboral en Ecuador. Revista Acción Psicológica 14(2), 225-239.

Roa, C. (2019). Bienestar Laboral. Bogotá, Colombia: Trabajo especial de grado de la Universidad de América para optar al título de Especialista en Gerencia del Talento Humano.

Sanchez, I. (2019). Inteligencia Emocional y el rendimiento laboral de los colaboradores del complejo Comercial Unicachi, Comas 2019. Lima, Perú: Trabajo especial de grado de la Universidad César Vallejo para optar al título de Licenciatura en Administración.

Veliz, A., Dörner, A., Soto, A., Reyes, J., \& Ganga, F. (2018). Inteligencia emocional y bienestar psicológico en profesionales de enfermería del Sur de Chile. Medisur $16(2)$. 
Yslado, R., Ramírez-Asís, E., García-Figueroa, M., \& Arquero, J. (2021). Clima Laboral y burnout en profesores universitarios. Revista Electrónica Interuniversitaria de Formación del Profesorado, 2483), 101-114.

Zhañay, J. (2017). Estrés laboral y clima organizacional en el personal de enfermería de un hospital público. Quito, Ecuador: Trabajo especial de grado de la Universidad Central del Ecuador para optar por el título de Magister en Seguridad y Salud Laboral. 
El artículo que se publica es de exclusiva responsabilidad de los autores y no necesariamente reflejan el pensamiento de la Revista Alfa Publicaciones.

\section{Ciencia}

El artículo queda en propiedad de la revista y, por tanto, su publicación parcial y/o total en otro medio tiene que ser autorizado por el director de la Revista Alfa Publicaciones.
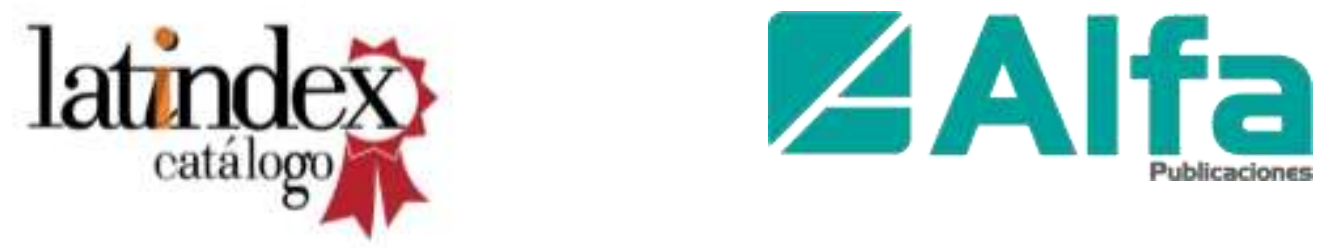

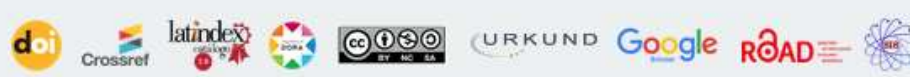
DLatinREV

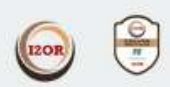

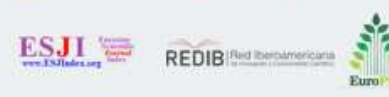
$\underbrace{}_{\text {wizdom.ai }} \bigodot_{\text {OpenAlRE }}^{\oplus}$

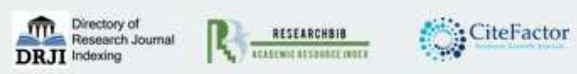

